\title{
THE SIGNIFICANCE OF LEFT AXIS DEVIATION IN HEART DISEASE OF THE AFRICAN
}

\author{
BY \\ L. SCHAMROTH AND D. BLUMSOHN \\ From the Baragwanath Hospital and University of the Witwatersrand, Johannesburg \\ Received December 5, 1960
}

The pattern of heart disease in the African (Negro) differs from that in the White (Caucasian) in two notable respects-the virtual absence of myocardial infarction $(0.4 \%$, Schwartz et al., 1958; $1.4 \%$, Laurie et al., 1960) and the presence of a high proportion of cases of obscure æatiology $(37.5 \%$, Schwartz et al., 1958; 31.5\%, Laurie et al., 1960). The latter have been referred to by various authors as idiopathic heart disease, nutritional heart disease (Gillanders, 1951), cardiovascular collagenosis with parietal endocardial thrombosis (Becker et al., 1953) and cryptogenic heart disease (Schwartz et al., 1958). Cryptogenic cardiomyopathy would appear to be a better term for this group of cases. They present with congestive cardiac failure, moderate to gross œdema, generalized cardiomegaly, gallop rhythm, occasional systolic murmurs, a hypokinetic circulation, and an inert heart on fluoroscopy-a presentation not unlike myxœedematous heart disease or pericardial effusion. There is no evidence of ischæmic, hypertensive, rheumatic, or pulmonary heart disease. Pathological examination reveals a basic pattern of dilatation of all chambers of the heart, moderate myocardial hypertrophy, and a patchy interstitial or endocardial fibrosis which is seldom marked. Antemortem intramural thrombi are commonly found in all chambers. Widespread endomyocardial fibrosis found in other parts of Africa (Bedford and Konstam, 1946; Ball et al., 1954; Davies and Ball, 1955) is extremely rare in South Africa. Some of these cases have been observed in this hospital (Seftel and Susser, 1960) during late pregnancy, the puerperium, and the lactational period-post-partum heart disease. This pattern is similar to that described as post-partal heart disease (Malvin, 1947), post-partum myocardosis (Vilter and McKee, 1953), and idiopathic myocardial degeneration of the puerperium (Gouley et al., 1937), by observers in America.

Recent work (Grant, 1956; Davies and Evans, 1960) has indicated that significant left axis deviation is not due to left ventricular hypertrophy per se, but to a conduction defect involving the anterior superior division of the left bundle branch. This conduction defect is most frequently the result of myocardial infarction or diseases associated with fibrosis of the left ventricle. The left bundle branch divides into a number of small branches almost immediately after leaving the bundle of His. These branches divide into two major sweeps or radiations that innervate the anterior superior aspect-the anterior superior division-and the posterior inferior aspect of the left ventricle - the posterior inferior division (Fig. 1). Excitation and conduction normally progress simultaneously through the fibres of both divisions. If, however, interruption of conduction occurs in the fibres of the anterior superior division, activation of the ventricular walls will occur chiefly through the posterior inferior division without necessarily causing prolongation of the QRS complex. As the principal excitation via the posterior inferior division is terminally upwards and to the left (arrow 2, Fig. 1), the dominant vector will be upward and to the left resulting in left axis deviation.

The possibility that the patchy fibrosis found in the cases of cryptogenic cardiomyopathy of the 


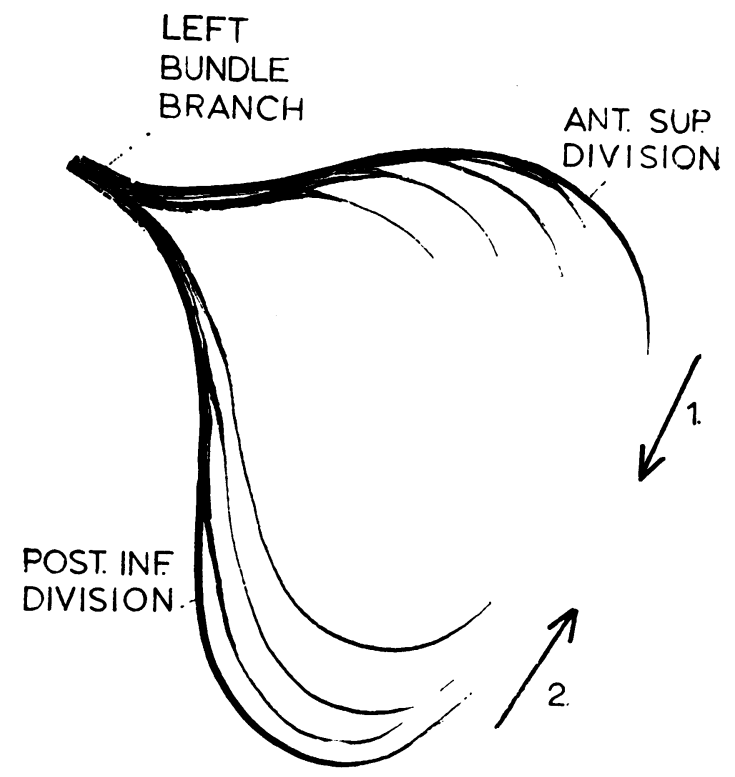

FIG. 1.-Semi-diagrammatic representation of the left bundle branch. Ant. Sup.=Anterior superior; Post. Inf. $=$ Posterior inferior. Arrows 1 and 2 indicate direction of terminal vector forces of each bundle branch.

African would likewise result in significant left axis deviation prompted this investigation. As the presentation of cryptogenic cardiomyopathy may mimic myxœdema or pericardial effusion, and, when systolic murmurs are pronounced, mitral regurgitation, these diseases were analysed for the occurrence of left axis deviation. Furthermore, as myocardial infarction and chronic coronary heart disease are the chief causes of left axis deviation in the White, and as these conditions are extremely uncommon in the African, the disease spectrum of left axis deviation in the African was also studied.

\section{MATERIAL AND Methods}

The mean manifest frontal plane QRS and T wave axes (ÂQRS and ÂT) were studied in 256 persons at Baragwanath Hospital, all of pure African (Negro) descent. These included 48 patients with cryptogenic cardiomyopathy (4 with left bundle-branch block), who were compared with 70 patients with hypertension, 19 with mitral regurgitation, 4 with myxœdema, and 5 with pericardial effusion. The hypertensive patients were selected on the basis of diastolic pressures of over $100 \mathrm{~mm}$. Hg. All were in-patients with clinical, electrocardiographic, and radiological manifestations of advanced hypertensive heart disease. Thirty-five healthy African nurses and 60 healthy African male labourers attached to the hospital were selected as normal controls. Their ages ranged from 18 to 67 years (men 23 to 67 years; women 18 to 27 years). These subjects lived on the hospital premises and were given a standard staple balanced diet.

In addition, 76 consecutive instances of left axis deviation were examined and the disease spectrum analysed. This category included all the instances of left axis deviation in the aforementioned groups as well as a miscellaneous group consisting of arteriosclerosis, constrictive pericarditis, persistent A-V communis, coarctation of the aorta, and aortic and ventricular aneurysms.

Due to the persistent bed shortage in this hospital, most patients admitted to the wards are severely ill and usually in an advanced stage of their illness. 
All electrocardiograms were recorded by a direct writing electrocardiograph with the subjects in the supine position.

Axes were plotted on Bayley's triaxial reference system with the addition of the unipolar limb lead axes forming a hexaxial reference system (Fig. 2 and 3).

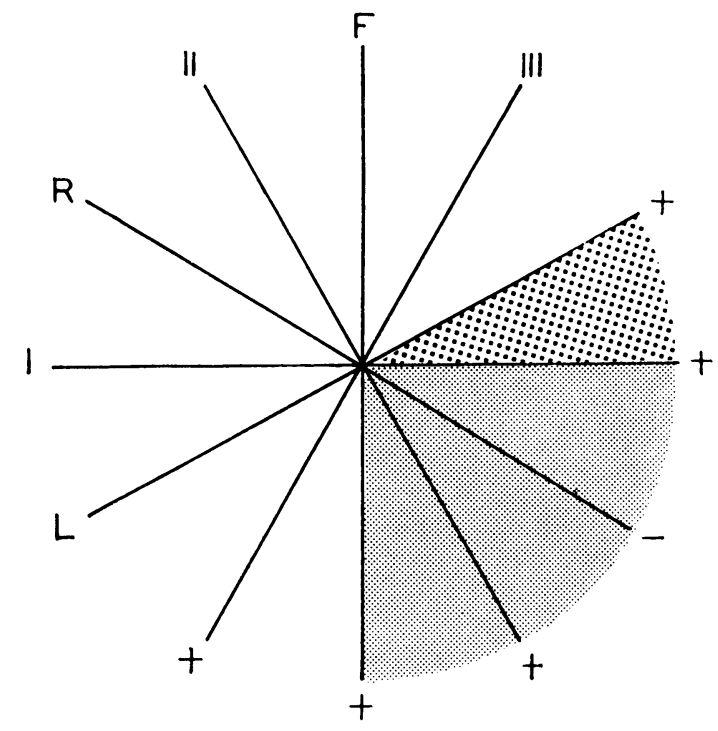

FIG. 2.-Hexaxial reference system, showing normal range of ÂQRS.

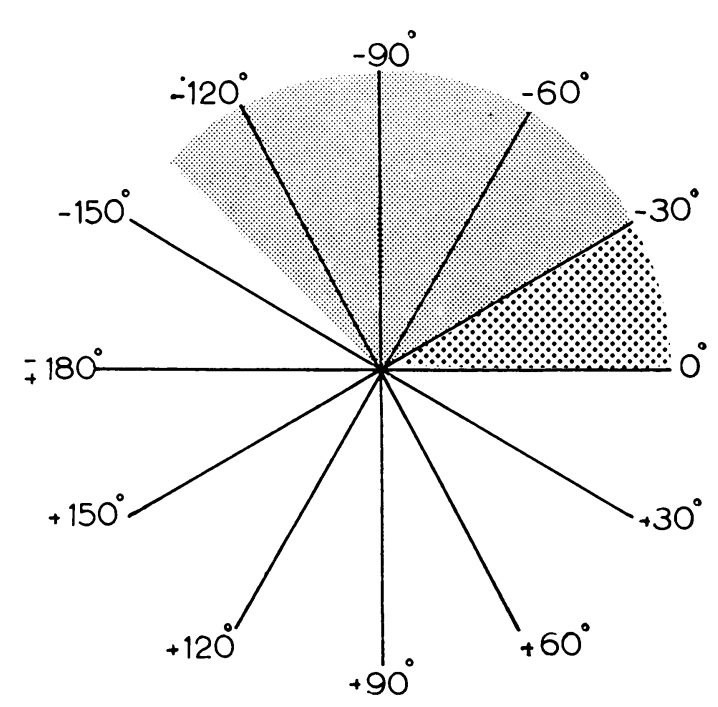

FIG. 3.- Hexaxial reference system, showing range of slight left axis deviation (light shading) and significant left axis deviation (dark shading).

The normal range of ÂQRS is usually between 0 and $+90^{\circ}$. Axes between 0 and $-29^{\circ}$ may occasionally be found in the normal subject, especially during pregnancy or in those with much obesity or a stocky build. It may also be associated with severe ascites or abdominal distension. However, axes in this zone should be viewed with suspicion as they are frequently due to organic heart disease. Grant (1956) considers an ÂQRS more leftward than $-15^{\circ}$ as significant. The criteria for significant left axis deviation in this paper are stringently defined as a leftward shift of $-30^{\circ}$ or greater. This implies dominant $S$ waves in leads II and III and usually dominant $R$ waves in standard lead I (Fig. 8). Axes in the zone of 0 to $-29^{\circ}$ are referred to as slight left axis deviation (Fig. 3). An ÂQRS-T angle spread greater than $60^{\circ}$ is regarded as abnormal.

\section{RESULTS}

Normal Subjects. Only one of the 95 normal controls had left axis deviation. This was a man aged 42 in whom ÂQRS was $-30^{\circ}$. The $T$ wave axis in this subject was normal and there were no other electrocardiographic abnormalities. ÂQRS in the remaining subjects ranged from 0 to $+90^{\circ}$ with a mean of $+47^{\circ}$ (Fig. 4). Age and sex were without influence on the distribution of ÂQRS. (It should be noted that the youngest subject was 18 years old.) Four men had a QRS-T spread of $60^{\circ}$ and the subject with left axis deviation referred to above had a QRS-T spread of $75^{\circ}$. The remainder had QRS-T angles of less than $60^{\circ}$. The electrocardiogram in one woman showed the Wenckebach phenomenon. There were no other cardiographic abnormalities in our cases.

These results are similar to those reported in the White (Caucasian) ethnic group. Thus Davies and Evans (1960) found that 3 out of 200 normal subjects had left axis deviation of ÂQRS, all three cases being over 40 years of age. Of 558 cases studied by Zao et al. (1958) ÂQRS was located between 0 and $+90^{\circ}$ in all except one in whom it was $-15^{\circ}$. The QRS-T angles ranged from 0 to $45^{\circ}$. 
Cryptogenic Cardiomyopathy. (a) Cases without QRS Prolongation. Of the 44 patients, significant left axis deviation was found in $20(46 \%)$. Slight left axis deviation was present in $10(23 \%)$. Normal axes were found in $11(25 \%)$, and the remaining 3, all of whom had gross functional tricuspid regurgitation, showed right axis deviation (Fig. 5).

The electrocardiograms also showed other abnormalities. In 31 cases the $\mathbf{T}$ waves were inverted over V5 and V6. In 26 cases the QRS-T angle exceeded $60^{\circ}$. In addition, there were instances of uni- and multifocal ventricular extrasystoles, first degree A-V block, and atrial fibrillation. There were no instances of $U$ wave inversion, sharp-angled ST-T junctions, or plane depression of the S-T segments. The T waves were not pointed or symmetrical.

(b) Cases with QRS Prolongation. There were 4 cases with left bundle-branch block. Two of these had terminal vectors showing significant left axis deviation $\left(-75^{\circ}\right.$ and $\left.-60^{\circ}\right)$ (Fig. 9). Moreover, the terminal vectors were more leftward than the initial vectors indicating that left axis deviation was present before the development of left bundle-branch block (Grant, 1957). In both these cases the angle between initial and terminal vectors did not exceed $60^{\circ}$. In the third case both initial and terminal vectors were normally directed.

The fourth case also showed normally directed initial and terminal vectors (Fig. 10A). However, 5 months later the terminal vector had shifted to the left, showing significant left axis deviation $\left(-80^{\circ}\right)$ : Fig. 10B); the initial vector remained normal $\left(+70^{\circ}\right)$. This spread of $150^{\circ}$ indicates a pattern suggestive of peri-infarction block (vide infra). This patient denied any chest pain and there was no evidence of myocardial infarction in the electrocardiogram.

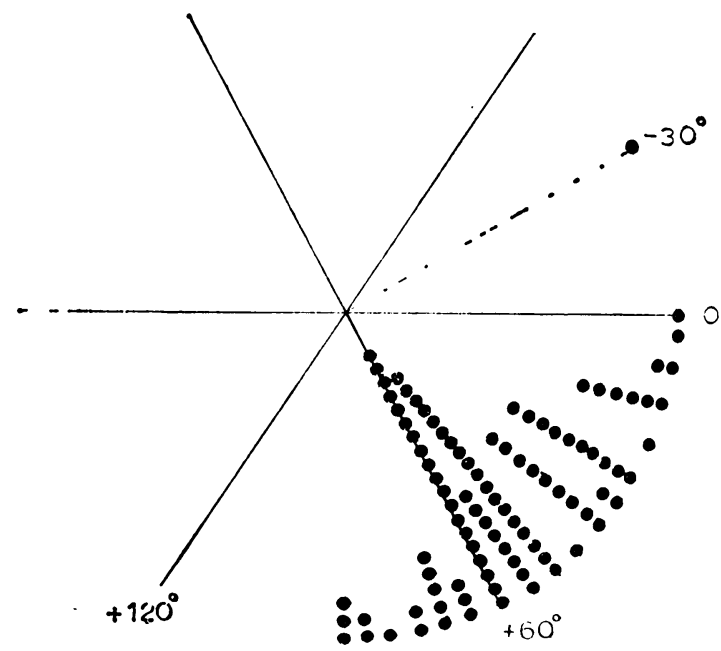

FIG. 4.--ÂQRS distribution in normal African subjects.

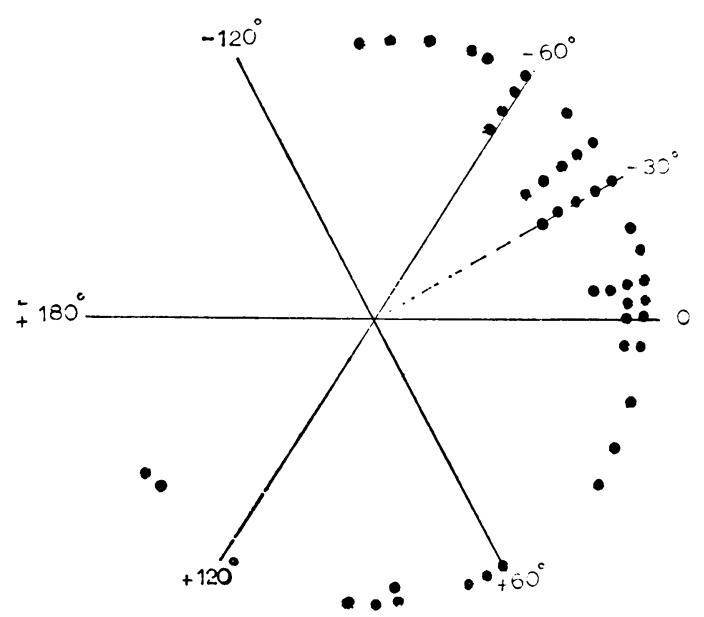

FIG. 5.-ÂQRS distribution in cases with cryptogenic cardiomyopathy.

Hypertension. Significant left axis deviation was found in 12 of 70 patients $(17 \%)$. Nearly all were examples of long-standing hypertensive disease with complicating factors such as cardiac failure, diabetes mellitus, or renal or cerebral vascular disease. Slight left axis deviation was found in $19(27 \%)$. Normal axes were present in $39(56 \%)$ (Fig. 6).

The average age for those with left axis deviation was 58 years and for those with normal axes 45 years. The categories with slight or significant left axis deviation had a higher percentage of patients with marked elevation of the diastolic pressures than the group with normal axes (Table I).

Mitral Regurgitation. Of these 19 patients none showed significant or even slight left axis deviation. The tendency was for a deviation of ÂQRS to the right or frank right axis deviation (greater than $+90^{\circ}$ ) (Fig. 7). The QRS-T angles ranged from 0 to $50^{\circ}$. $\mathrm{T}$ waves were tall and upright in leads V5 and V6 in all cases. 
TABLE I

Relation Between ÂQRS and Diastolic Blood Pressure

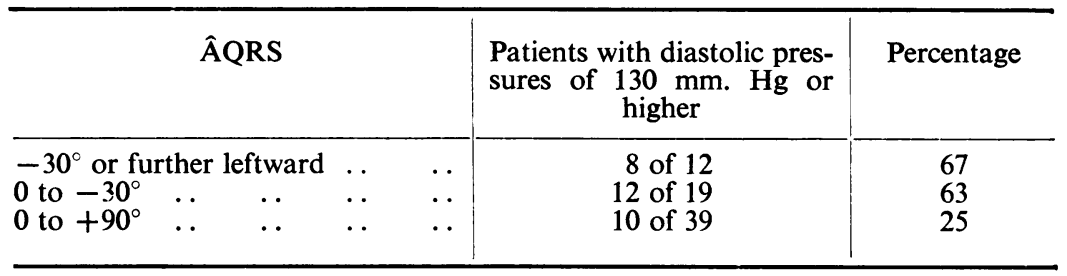

Pericardial Effusion and Myxadema. None of these cases showed left axis deviation. ÂQRS in 5 cases of pericardial effusion ranged from $+40^{\circ}$ to $+120^{\circ}$, and in 4 cases of myxœedema from $+15^{\circ}$ to $+75^{\circ}$.

\section{Study of Consecutive Cases of Left Axis Deviation}

Analysis of 42 cases with significant, and 34 with slight, left axis deviation revealed the distribution shown in Table II. This did not include the one normal subject whose ÂQRS was $-30^{\circ}$. All these cases showed, in addition, other electrocardiographic abnormalities either in the patterns or in rhythm. No patterns of myocardial infarction were observed.

TABLE II

Left Axis Deviation in Various Diseases

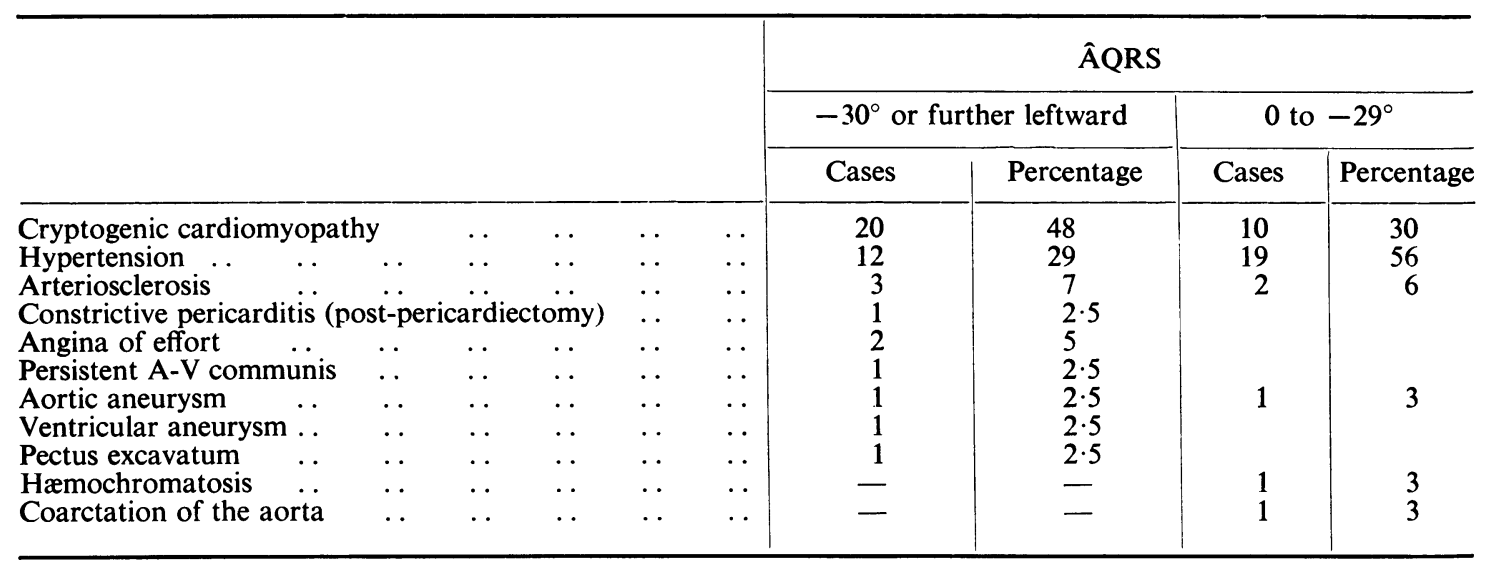

\section{Discussion}

The principal conditions that may cause conduction defects in the anterior superior division of the left bundle branch and result in left axis deviation are myocardial infarction, advanced left ventricular hypertrophy, and chronic coronary heart disease.

Myocardial Infarction. In the White (Caucasian) ethnic group antero-lateral infarction is an important cause of left axis deviation, and is referred to as peri-infarction block. Thus, Grant (1956) found infarction in 54 of $131(41 \%)$ consecutive cases of left axis deviation. Of these, the infarction was located in the antero-lateral aspect of the left ventricle in 34. Davies and Evans (1960) found left axis deviation in 32 of $200(16 \%)$ patients with myocardial infarction. The left axis deviation of peri-infarction block may be distinguished from that due to other causes in the following manner: in myocardial infarction the initial vector (initial $0.04 \mathrm{sec}$. of the QRS complex) 


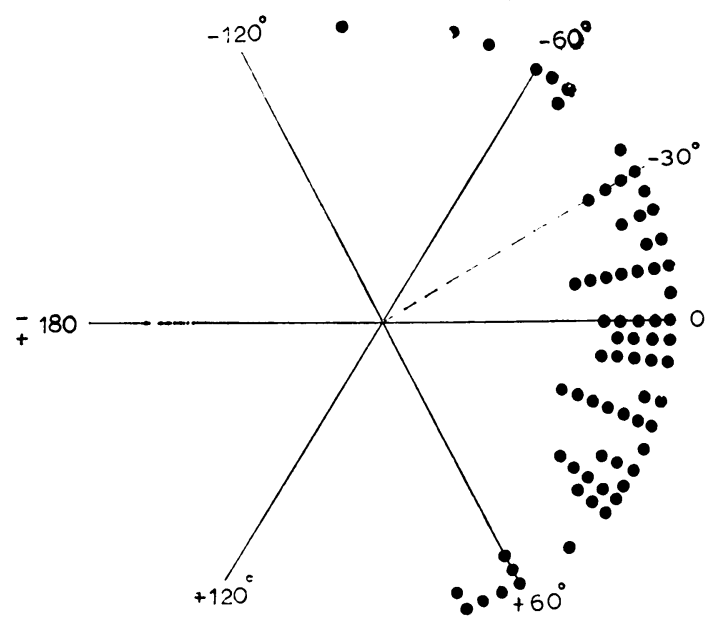

FIG. 6.-ÂQRS distribution in cases with hypertension.

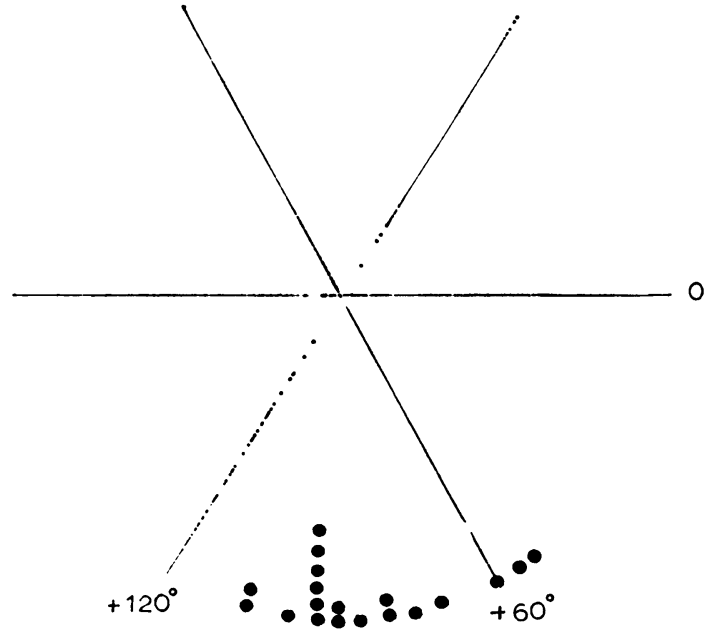

FIG. 7.-ÂQRS distribution in cases with mitral regurgitation.

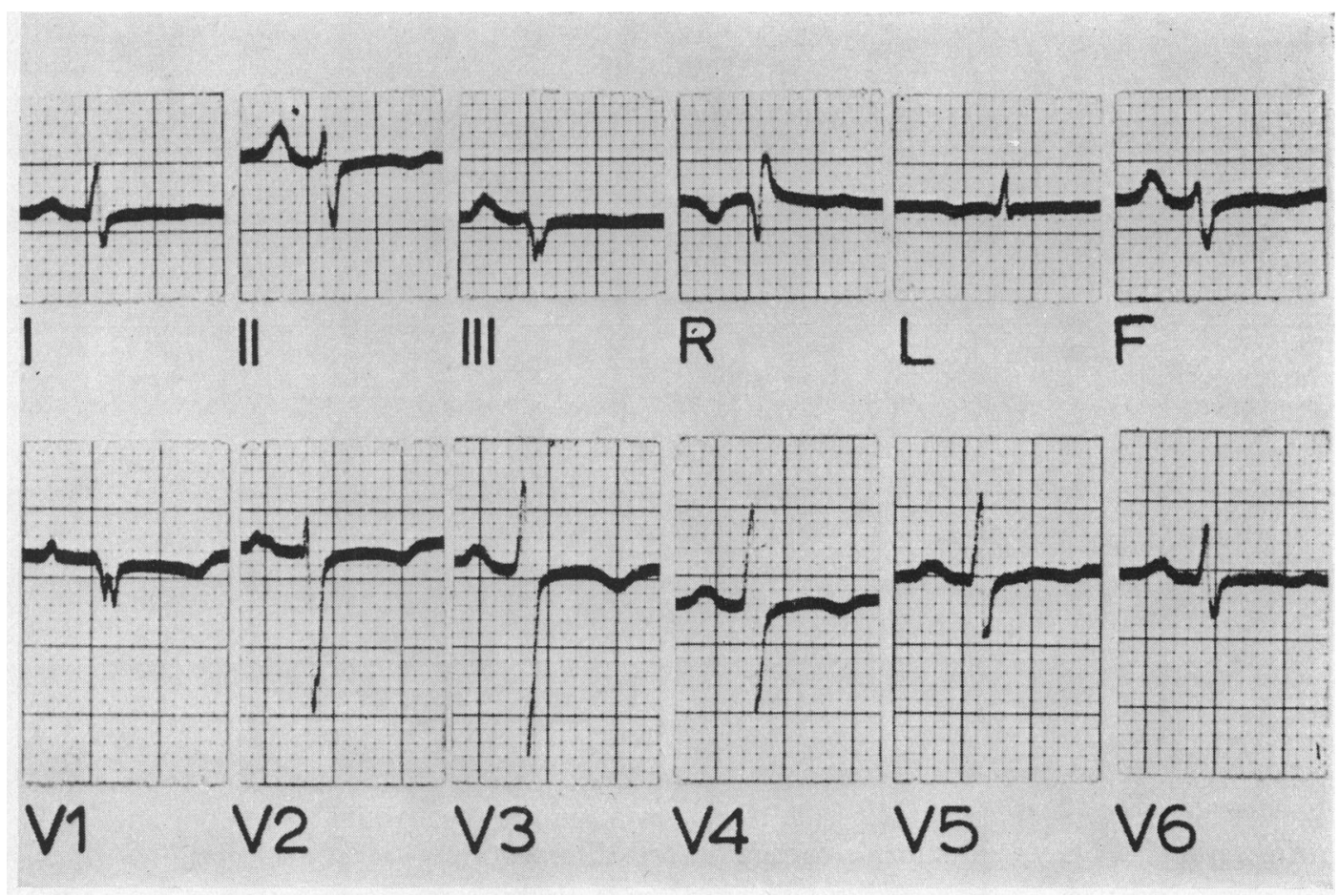

FIG. 8.-Electrocardiogram showing significant left axis deviation in post-partum cardiomyopathy.

is directed away from the infarcted area; in the case of antero-lateral infarction the initial vector would thus be downward and to the right. The terminal vector (terminal $0.04 \mathrm{sec}$.) would, as discussed above, be directed upwards and to the left. Thus in peri-infarction block the angle of spread between the initial and terminal vectors usually exceeds $100^{\circ}$, whereas in other causes of left axis deviation it rarely exceeds $60^{\circ}$ (Grant, 1957). 


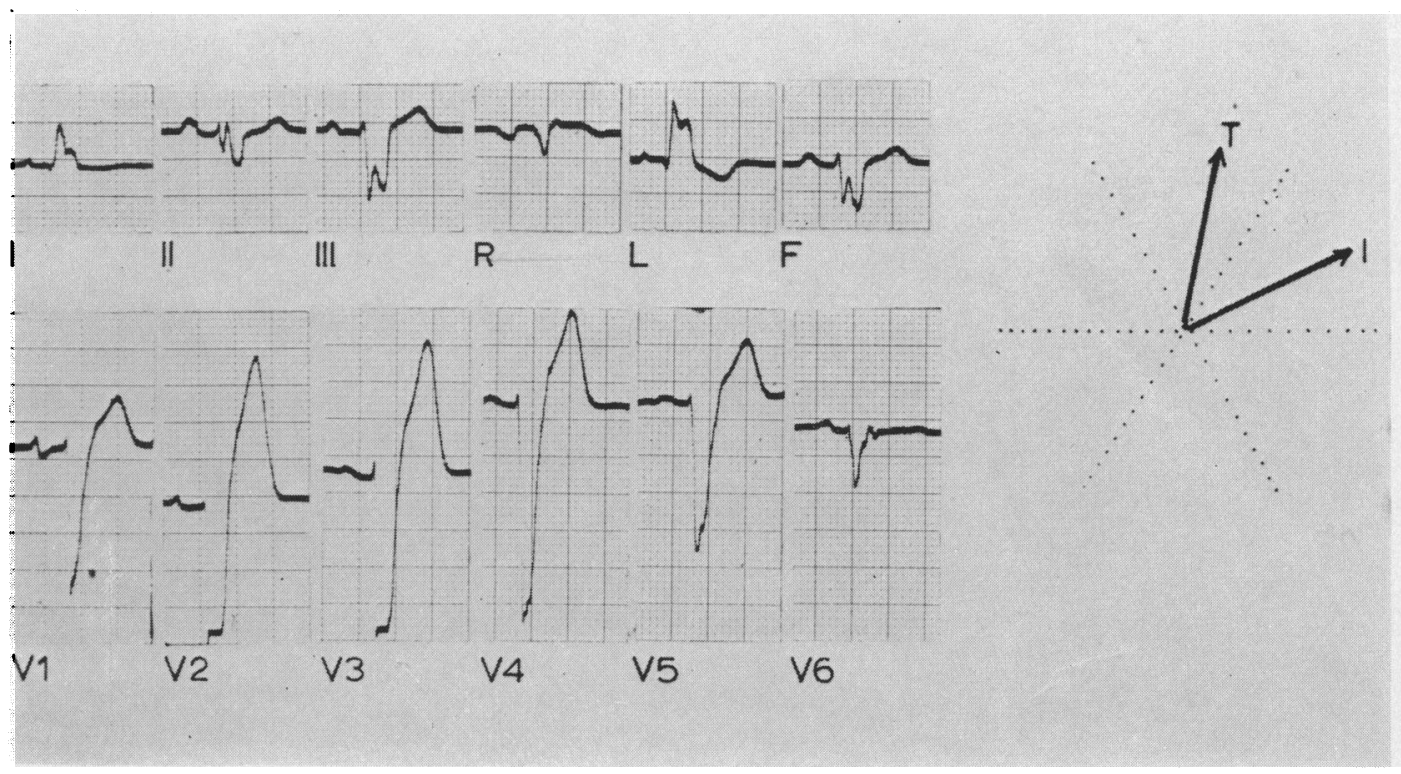

FIG. 9.-Electrocardiogram in a case of cryptogenic cardiomyopathy, showing left bundle-branch block, and left axis deviation of both initial (I) and terminal (T) vectors. The terminal vector is further leftward than the initial vector.

By virtue of the fact that myocardial infarction is extremely rare or virtually absent in the African population, peri-infarction block does not constitute any significant cause of left axis deviation. Indeed, we did not encounter one case of myocardial infarction in the 256 electrocardiograms examined. Furthermore, we found only one example of peri-infarction block in all our cases of left axis deviation: this was in a patient with cryptogenic cardiomyopathy. Thus, as indicated by Davies and Evans (1960) peri-infarction block does not necessarily connote infarction in its literal meaning, as it may occur with fibrosis alone. The term should therefore be retained as an electrocardiographic conception alone.

Advanced Left Ventricular Hypertrophy. This is sometimes associated with left axis deviation. However, as Grant (1957) has indicated "it is not the hypertrophy itself that produces the left axis deviation, because the majority of cases of even severe left ventricular hypertrophy have a normally directed mean QRS axis. Probably the myocardial fibrosis which so often accompanies marked left ventricular hypertrophy is responsible, by involving certain peripheral parts of the left ventricular conducting system." This condition is analogous to what has previously been called "parietal block" (Segers et al., 1947; Alzamora-Castro et al., 1953).

Davies and Evans (1960) found left axis deviation in only 8 of 50 patients with severe hypertension, 7 of whom either had chest pain or electrocardiographic evidence of myocardial infarction. Likewise, Grant (1956), after the exclusion of all cases of myocardial infarction, noted that left axis deviation did not necessarily correlate with the heart weight or body build. Nevertheless, the incidence of left axis deviation was higher among cases with hypertrophied left ventricles than among those with normal heart weights, and he attributed this to the myocardial fibrosis that accompanies severe left ventricular hypertrophy.

In the present series 44 per cent of patients with hypertension showed left axis deviation. However, it should be stressed that these were all advanced or long-standing cases of hypertension with cardiac failure, marked retinitis, and complicated in the majority of instances by renal disease, diabetes mellitus, or cerebral vascular accidents. Indeed, in those cases where hypertension was 

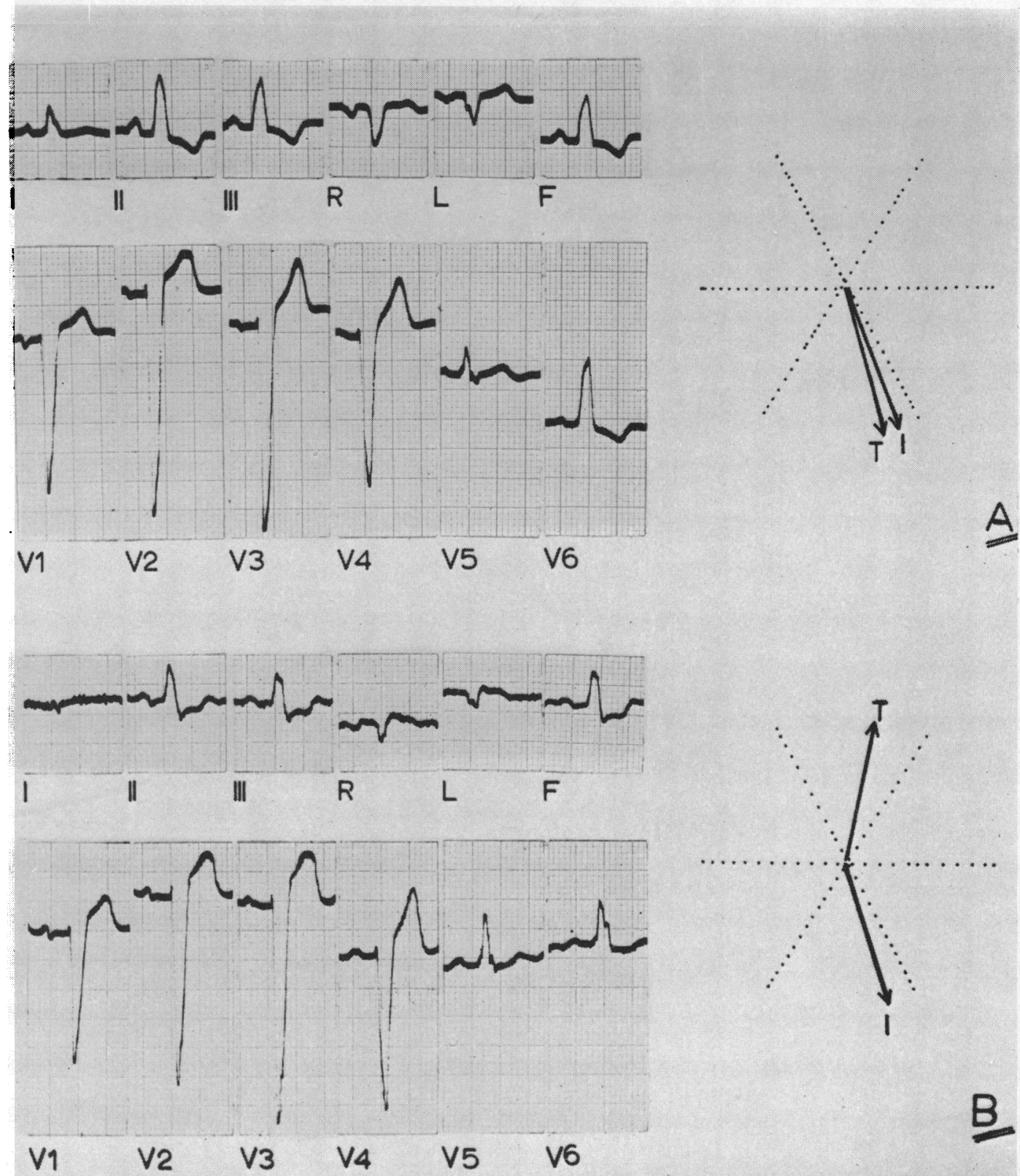

FIG. 10.-Electrocardiograms of patient with cryptogenic cardiomyopathy. (A) Left bundle-branch block with normally directed initial (I) and terminal (T) vectors. (B) Five months later-leftward shift of the terminal vector, suggestive of peri-infarction block.

the only or dominant feature, the axis usually fell within the normal range. The group with significant left axis deviation had a higher percentage of patients with marked elevation of the diastolic pressure than the group with normal axes (Table I). However, this is most unlikely to be due to the raised diastolic pressure per se, otherwise it could reasonably be anticipated that no cases with 
much rise of diastolic pressure would have normally directed axes. Rather, it is the structural change accompanying the raised diastolic pressure that is more likely to be the cause.

Chronic Coronary Heart Disease. This also is a cause of left axis deviation by virtue of the fibrosis it produces. This is probably the cause of left axis deviation in the elderly patient where myocardial infarction and long-standing hypertension can be excluded. As with myocardial infarction, chronic coronary heart disease is rare among the African population. Thus we found 3 patients (aged 103, 65 , and 49 years) where significant left axis deviation could possibly be attributed to chronic coronary heart disease. Significant left axis deviation was also found in 2 patients (aged 65 and 50 years) who presented with angina of effort. However, in our normal controls there were 12 men over 50 years of age who did not show left axis deviation. Of the 200 normal subjects examined by Davies and Evans (1960), the three with left axis deviation were all over 40 years of age. We had one subject among our normal controls, a man aged 42, with ÂQRS of $-30^{\circ}$; and although chronic coronary heart disease may have been a factor, we doubt this because, apart from the rarity of chronic coronary heart disease in this population, he showed none of its stigmata. It may be possible, as suggested by Manning (quoted by Grant, 1958) that this may be an example of a rare congenital malformation of the distal radicle of the left bundle branch. Subsequent to their investigations, Davies and Evans (1960) found left axis deviation in four young subjects with no evidence of heart disease in whom this mechanism of congenital agenesis was postulated.

Cardiomyopathy. Davies and Evans (1960) found left axis deviation in $16(64 \%)$ of 25 cases with obscure cardiomyopathy, They also found it in a number of cases of familial cardiomegaly, Friedreich's disease, cardiac amyloidosis, myotonia atrophica, and hæmochromatosis.

In our group of cryptogenic cardiomyopathy of the African, 46 per cent had significant left axis deviation, and 23 per cent had slight left axis deviation. Thus 69 per cent showed some degree of left axis deviation. The cases of cryptogenic cardiomyopathy included 5 with postpartum heart disease whose axes were $-50,-30,-10,+10$, and +30 degrees. Autopsy in one of these (ÂQRS $\left.-30^{\circ}\right)$ revealed a moderately enlarged heart (440 g.) with dilatation affecting principally the left ventricle. The left ventricular musculature was not unduly thickened, and measured $1.3 \mathrm{~cm}$. at the outflow tract. There were three well-marked plaques of fibrous endocardial thickening in the left ventricle. One at the apex measured $1 \mathrm{~cm}$. in diameter. Another was situated over the antero-lateral aspect halfway up the wall of the left ventricle to the left of the septum; it measured $0.3 \times 1 \mathrm{~cm}$., and extended laterally to the left for about $1 \mathrm{~cm}$. A third small focus, which measured $0.4 \mathrm{~cm}$. was situated immediately below the aortic valve.

Analysis of the group comprising all consecutive cases of left axis deviation irrespective of cause revealed that cryptogenic cardiomyopathy was by far the commonest cause, viz., 48 per cent. The mechanism of left axis deviation in these cases is probably similar to that operating in myocardial infarction, chronic coronary heart disease, and advanced left ventricular hypertrophy, viz., involvement of the left bundle branch by fibrosis.

Gross endomyocardial fibrosis is extremely rare in South Africa. Nevertheless, it could reasonably be anticipated that the same mechanism would be operative in this condition, and that left axis deviation would likewise be a notable feature. Although no specific reference to axis deviation is mentioned, it is interesting to note that in the one electrocardiogram of endomyocardial fibrosis published by Turner and Manson-Bahr (1960) the ÂQS is $-40^{\circ}$.

None of the cases of mitral regurgitation, pericardial effusion, and myxœdema showed any tendency whatsoever to left axis deviation. Thus the presence of left axis deviation would appear to be of considerable help in excluding these conditions. Further differentiating features are the low voltage in pericardial effusion and myxœdema, and the fact that T waves in V5 and V6 were always upright in mitral regurgitation, whereas in the majority of cases of cryptogenic cardiomyopathy they were inverted.

Williams and Somers (1960) in a study of electrocardiographic abnormalities in endomyocardial fibrosis describe non-specific changes without referring to changes in ÂQRS. They stress the 
difficulty of distinguishing endomyocardial fibrosis from chronic rheumatic mitral disease and pericarditis, a difficulty previously noted by Ball et al. (1954). However, as with cryptogenic cardiomyopathy the presence of left axis deviation may well be a distinguishing feature and warrants further investigation.

It is apparent that in the local African cryptogenic cardiomyopathy is the commonest cause of left axis deviation. This is in contrast to the Caucasian where myocardial infarction and coronary heart disease are the chief causes of left axis deviation. Thus in an African patient presenting with cardiomegaly in the absence of advanced hypertension, the presence of left axis deviation is suggestive of cryptogenic cardiomyopathy.

It should be stressed that left axis deviation is not specific to any of the disease in which it occurs, but merely represents a conduction defect most commonly due to non-specific fibrosis. In the absence of myocardial infarction, coronary heart disease, and advanced hypertension, the presence of left axis deviation, irrespective of race and particularly in the younger subject is a pointer to a cardiomyopathy.

\section{SUMMARY}

The mean manifest frontal plane QRS and T wave axes (ÂQRS and ÂT) were studied in cases of cryptogenic cardiomyopathy in the African (Negro) and compared with cases of hypertension, mitral regurgitation, pericardial effusion, and myxœdema.

Electrocardiograms of healthy African male orderlies and nurses attached to the hospital were used as normal controls. With one exception, ÂQRS in the normal controls was located between 0 and $+90^{\circ}$. Cases with cryptogenic cardiomyopathy showed a significantly high incidence of left axis deviation and formed the major ætiological group of all causes of left axis deviation in the African.

Significant left axis deviation $\left(-30^{\circ}\right.$ or further leftward) was found only in those hypertensive patients who had advanced disease with complicating factors.

The presence of left axis deviation may help to distinguish cryptogenic cardiomyopathy from pericardial effusion, myxœdema, or mitral regurgitation. In the absence of coronary heart disease and advanced hypertension the presence of left axis deviation is a pointer to a cardiomyopathy.

We wish to express our thanks to Dr. I. Frack, Superintendent of Baragwanath Hospital for permission to publish these cases, Dr. E. Kahn for his helpful criticism of the manuscript, Dr. C. Isaacson for the pathological report, and Mr. A. Shevitz of the Photographic Dept. of the University of the Witwatersrand for the photographic reproductions.

\section{REFERENCES}

Alzamora-Castro, V., Abugattas, R., Rubio, C., Bouroncle, J., Zapata, C., Santa-Maria, E., Battilana, G., Binder, T., Subiria, R., and Paredes, D. (1953). Circulation, 7, 108.

Ball, J. D., Davies, J. N. P., and Williams, A. W. (1954). Lancet, 1, 1049.

Becker, B. J. P., Chatgidakis, C. B., and van Lingen, B. (1953). Circulation, 7, 345.

Bedford, D. E., and Konstam, G. L. S. (1946). Brit. Heart J., 8, 236

Davies, H., and Evans, W. (1960). Brit. Heart J., 22, 551.

Davies, J. N. P., and Ball, J. D. (1955). Brit. Heart J., 17, 337.

Gillanders, A. D. (1951). Brit. Heart J., 13, 177.

Gouley, B. A., McMillan, T. M., and Bellet, S. (1937). Amer. J. med. Sci., 194, 185.

Grant, R. P. (1956). Circulation, 14, 233.

- (1957). Clinical Electrocardiography. McGraw-Hill Book Co. Inc. (New York).

- (1958). Third World Congress of Cardiology. Brussels.

Laurie, W., Woods, J. D., and Roach, G. (1960). Amer. J. Cardiol., 5, 48.

Melvin, J. P. (1947). Ann. intern. Med., 27, 596.

Schwartz, M. B., Schamroth, L., and Seftel, H. C. (1958). Med. Proc., 4, 275.

Seftel, H. C., and Susser, M. (1961). Brit. Heart J. 23, 43.

Segers, M., Vastesaeger, M., and Denolin, H. (1947). Acta. Cardiol., 2, 166.

Turner, P. P., and Manson-Bahr, P. E. C. (1960). Brit. Heart J., 22, 305.

Vilter, R. W., and McKee, E. E. (1943). Ohio State med. J., 39, 142.

Williams, A. W., and Somers, K. (1960). Brit. Heart J., 22, 311.

Zao, Z. Z., Hermann, G. R., and Hejtmancik, M. R. (1958). Amer. Heart J., 56, 65. 